\title{
Ejercicio físico y COVID-19: la importancia de mantenernos activos
}

\author{
LUZ MARÍA TRUJILLO G.***, ASTRID VON OETINGER G.*** y DANIELA GARCÍA L.*****
}

\section{Physical exercise and COVID-19: the importance of keeping us active}

COVID-19 pandemic outbreak, as well as strategies aiming to control it, have created an unfavorable environment to keep up with daily life activities. No physical activity instructions nor suggestions have been given by regulatory estates (government, education, or labor). Exercise and physical activity benefit regarding immune system strengtheningn have been widely documented, which is why the aim of this study is to analyze existing literature of exercise and physical activities through COVID-19 pandemic outbreak. Material and Methods: Narrative bibliographic study including existing evidence on this subject. Conclusions: Analyzed evidence support the importance of physical activity during pandemic times. Daily physical activity plays a fundamental role in the fight against COVID-19, especially in the most vulnerable population.

Key words: COVID 19; Exercise; Physical Activity; Vulnerable population; Immune system.

\section{Resumen}

La pandemia desatada por el COVID-19 y las estrategias implementadas para intentar controlarla, han creado un ambiente poco propicio para mantener las actividades de la vida diaria. En relación con la actividad fisica no se han entregado medidas ni recomendaciones por parte de los estamentos reguladores (gubernamentales, educacionales, laborales). Los beneficios de la actividad fisica y del ejercicio en el fortalecimiento del sistema inmune han sido ampliamente documentados. Es por esto que el propósito de esta revisión es analizar la evidencia científica disponible sobre ejercicio y actividad fisica en tiempos de pandemia por COVID-19. Material y Métodos: Revisión de tipo bibliográfico narrativo que incluyó los artículos existentes en buscadores científicos sobre el tema. Conclusiones: La evidencia analizada respalda la importancia de realizar actividad física en tiempos de pandemia. La actividad fisica diaria juega un rol fundamental en la lucha contra el COVID-19, especialmente en la población más vulnerable.

Palabras claves: COVID 19; Ejercicio Físico; Población vulnerable; Sistema inmune.

\section{Introducción}

A nivel mundial se están viviendo tiempos muy difíciles tras la aparición de la enfermedad COVID-19, provocada por un virus de la familia coronavirus SARS-CoV-2. La Organización Mundial de la Salud (OMS) declaró, el 30 de enero, que el virus era considerado una amenaza para la salud pública por lo que se le clasificó como "Pandemia".
A principios de mayo, la cifra de contagiados a nivel mundial alcanzó las 3.760 .125 personas, dejando un saldo de 259.855 muertes a su haber. En Sudamérica, el número de casos en esa fecha ascendía a 116.299 en Brasil, 51.189 en Perú, 31.881 en Ecuador y 22.016 en Chile, con un número de muertes de 7.966, 1.444, 1.569 y 275 personas, respectivamente ${ }^{2}$. Estas cifras crecen día a día, ya que en esta región se registraron los primeros contagios a fines de febrero ${ }^{3}$. Hoy

\footnotetext{
Escuela de Kinesiología, Facultad de Ciencias de la Salud, Universidad de Las Américas. Santiago, Chile.

** Escuela de Kinesiología, Facultad de Odontología y Salud, Universidad Diego Portales. Santiago, Chile.

*** Escuela de Kinesiología, Facultad de Ciencias de la Salud, Universidad San Sebastián. Santiago, Chile.
} 
crece la incertidumbre del comportamiento de esta pandemia, ya que se vienen meses fríos por la llegada del invierno, lo que genera un ambiente propicio para la supervivencia del virus SARSCoV-2, así como también para la aparición de virus estacionales ${ }^{4}$ (Ej. Influenza, VRS), lo que sin duda hace aún más complejo el panorama.

Una de las estrategias más efectivas implementadas para mitigar la propagación del virus es el denominado "Aislamiento Social", en el que se limita el desplazamiento libre de las personas, se suspenden clases presenciales de escolares y universitarios, se promueve el teletrabajo, se decretan zonas de cuarentena parcial o total, y se restringen las actividades al aire libre.

Las personas se han confinado en sus casas, restringiendo su vida a cuatro paredes, y han visto un aumento considerable en su ingesta alimenticia y el tiempo de conductas sedentarias. Por otra parte, se ha mermado la posibilidad de realizar actividad física (AF), lo que sin duda es algo preocupante, ya que las cifras de inactividad física antes de la pandemia alcanzaban valores cercanos al $60 \%$ a nivel mundial ${ }^{5}$.

Por lo mismo, resulta alarmante que dentro de las medidas de autocuidado para hacerle frente al COVID-19 no se incluyan indicaciones para la realización de actividad física (AF). Sobre todo teniendo en consideración que existe evidencia suficiente que demuestra que la $\mathrm{AF}$ y el ejercicio son factores protectores ante enfermedades no transmisibles y generan un efecto positivo tanto para la salud mental como física ${ }^{6}$.

El objetivo de esta revisión es analizar la evidencia científica disponible respecto a la importancia de la actividad física durante esta pandemia a fin de: 1) Dar a conocer cómo afecta la inactividad física a nuestros sistemas fisiológicos; 2) Cómo se altera la capacidad inmunitaria por la inactividad física, pudiendo perjudicarnos frente a un posible contagio con SARS-CoV-2; 3) Indicar cuales son las estrategias recomendadas, en cuanto a actividad física y ejercicio físico, enunciadas por las diversas entidades internacionales y nacionales que debiesen de cumplir las personas para mantenerse saludables en situación de confinamiento.

\section{Material y Método}

El presente estudio es una revisión de tipo bibliográfica narrativa en la que se incluyeron 12 artículos científicos referentes al tema, con los siguientes términos MeSH: COVID-19, SARS-CoV-2, Actividad física, Sistema inmune,
Ejercicio físico. Se consideraron artículos de revisión sistemática (SR), artículos controlados randomizados (RTC), ensayos clínicos controlados (CCT), estudios observacionales (OS) y narraciones bibliográficas. Los artículos incluidos fueron publicados entre enero y mayo de 2020 . Los buscadores científicos utilizados fueron: PubMed, Cochrane, Science Direct y Medline, escritos en idioma inglés, español y chino.

\section{Comorbilidades y COVID- 19}

Respecto a las comorbilidades es importante destacar el estudio de Cascella M. et al. ${ }^{7}$, donde se evidencia que aproximadamente, el $49 \%$ de los casos críticos de COVID-19 poseen comorbilidades pre-existentes, como Enfermedades Cardiovasculares (ECV), Diabetes, Enfermedad Respiratoria crónica y Enfermedades Oncológicas. Lo anterior también se ha demostrado en otros artículos donde se suman condiciones de riesgo como el consumo de tabaco, hipertensión arterial y obesidad ${ }^{9}$. Zhonghua et al. ${ }^{9}$, evidenció que dichas comorbilidades aumentan la severidad y mortalidad $(10,5 \%$ con comorbilidad vs $0,9 \%$ sin comorbilidad) del COVID-19.

Por otra parte, en el estudio de Fishman et al. ${ }^{10}$,se demostró que en los pacientes inmunodeprimidos, el COVID-19 resulta una amenaza, puesto que determina un peor pronóstico en la recuperación. Cabe mencionar que todas las condiciones de salud previamente mencionadas tienen como factor común el aumento de citokinas inflamatorias lo que deriva en el debilitamiento del sistema inmune ${ }^{11}$.

\section{Obesidad y COVID-19}

En relación a la obesidad, se ha demostrado que tener exceso de peso es un factor de riesgo de desarrollar diferentes patologías, incluida el COVID-19. Es así que, en el estudio de Cao et al. ${ }^{12}$, se evidenció que aquellos sujetos con menor IMC tienen menor tasa de mortalidad por COVID-19.

Continuando con la asociación entre la obesidad y la mortalidad por COVID-19, nos parece interesante mencionar el artículo de Luzi et al. ${ }^{13}$, en que se estudió la relación existente entre influenza y obesidad y en la que se plantea cómo el comportamiento frente a ese virus puede servir de aprendizaje para enfrentar el virus COVID-19. Los autores concluyeron la importancia de fortalecer el sistema inmune, ya que está demostrado que en sujetos obesos y, más aún, en aquellos que además presentan diabetes, existe un aumento de mediadores inflamatorios, los que se asocia a mayor depresión inmunitaria ${ }^{13}$. Por otra parte, logran determinar por qué los sujetos con mayor 
IMC son más propensos al contagio, y definen 3 causales: 1) La eliminación viral (ejemplificando con la influenza) es hasta un 104\% mayor en sujetos obesos que en normopeso, lo que aumenta el ciclo viral y por ende se prolonga el período de contagio; 2) Se ha evidenciado aparición de nuevas cepas de virus con mayor replicación del ARN y carga viral aumentada; 3) Mayor carga viral en el aire espirado, lo que aumenta la probabilidad de contagiar a otros sujetos ${ }^{13}$. Los autores refuerzan la importancia de considerar estos factores en la pandemia del COVID-19, indicando a la población que favorezcan las medidas que permitan mantener un peso saludable, manejando la dieta y recomendando la práctica de ejercicio y actividad física a diario ${ }^{13}$.

\section{Espacios de confinamiento, inactividad física y sistemas corporales}

La pandemia que vivimos actualmente no tiene precedentes en cuanto a países afectados, población con medidas de cuarentena y restricciones aplicadas en los distintos países ${ }^{9}$. Los cambios que genera el mantenerse en espacios reducidos con poca posibilidad de desplazamiento sobre el sistema inmune ya han sido evidenciados, asociados al aumento de hormonas de estrés y otros mediadores ${ }^{10,14}$. En relación a esto, cabe destacar un estudio publicado en 2004 por Shimamiya y cols. que describe que el encierro genera efectos significativos sobre el sistema inmune, provocando estrés psíquico y fisiológico, que se evidencia a través del aumento de cortisol y la disminución de linfocitos. Lo anterior se asocia a un sistema inmune deprimido que es más susceptible a contraer enfermedades ${ }^{15}$.

La inactividad física se asocia a múltiples efectos perjudiciales para la salud, dentro de estos se incluyen; disminución de la capacidad aeróbica (reducción de aproximadamente un 7\% del $\dot{\mathrm{VO}}_{2}$ máx.), disminución de masa músculo-esquelética y disminución en la capacidad cognitiva ${ }^{16}$. A su vez, se ha determinado que la inactividad independiente de su causa genera una reducción en la funcionalidad cardiaca y un aumento en el riesgo de desarrollar enfermedad coronaria y de sufrir muerte súbita en el largo plazo ${ }^{17,18}$.

Por otra parte, la inactividad física es responsable de cambios a nivel metabólico provocando alteración de la señalización de la insulina, lo que genera a corto plazo un aumento de la resistencia insulínica y disminución de la lipólisis ${ }^{16}$. Los cambios en la lipólisis contribuyen al aumento de grasa visceral lo que se asocia a un mayor riesgo de desarrollar síndrome metabólico y a aumento del riesgo cardiovascular ${ }^{19}$.

Muchos de los pacientes que desarrollan COVID-19 son inactivos físicamente, no poseen una buena capacidad cardiorrespiratoria y presentan alteraciones metabólicas asociadas al sedentaris$\mathrm{mo}^{20}$. Afortunadamente, múltiples estudios han demostrado que la realización de ejercicio aeróbico mejora la respuesta ventilatoria en este tipo de pacientes (previamente inactivos y que cursaban enfermedades respiratorias). Destacándose principalmente los ejercicios aeróbicos que incluyen acondicionamiento de musculatura diafragmática, los que se consideran una potente herramienta clínica en la prevención de la disfunción diafragmática inducida por ventilación mecánica, y en la reducción de los problemas asociados al destete de pacientes expuestos a ventilación mecánica por largo tiempo ${ }^{21-23}$.

\section{COVID 19, ejercicio y sistema inmune}

Los beneficios fisiológicos que genera la actividad física y ejercicio (AF/ejercicio) sobre el sistema inmune han sido ampliamente investigados destacando dentro de sus efectos la mejora en la capacidad de eliminar patógenos y la disminución de incidencia de contraer distintas patologías ${ }^{24,25}$. Esto concuerda con los hallazgos de numerosos artículos en los que se ha demostrado que el ejercicio físico disminuye el riesgo de infección respiratoria y la severidad de esta incluso en personas que aún no presentan síntomas, logrando reducir morbilidad y mortalidad en afecciones como virus de Influenza, herpes simple 1 (HSV1) y rinovirus ${ }^{26-28}$. Esto ocurriría gracias a que la actividad física provoca un aumento en la inmunovigilancia contra patógenos infecciosos como los mencionados anteriormente ${ }^{29}$.

La AF genera efectos positivos sobre anticuerpos y glóbulos blancos, favoreciendo su formación y un aumento en su velocidad de circulación. Otra ventaja de la realización de AF, es que provoca un aumento en la temperatura corporal, lo que impide el crecimiento bacteriano ${ }^{15}$. Por otra parte, se ha demostrado que el ejercicio y la actividad física realizados de manera regular, disminuyen la secreción de catecolaminas y cortisol, lo que tiene un rol protector, disminuyendo el riesgo de contraer enfermedades ${ }^{24}$.

En el caso de las personas mayores, se ha determinado que el envejecimiento produce alteraciones perjudiciales sobre la inmunidad innata y adaptativa. Antecedente que toma relevancia al analizar las estadísticas del COVID-19, en 
las que se pone de manifiesto que el solo hecho de ser una persona mayor constituye un factor de riesgo para contraer y morir a causa de esta enfermedad $^{30}$. Jiménez-Pavón et al. ${ }^{31}$, mencionan la importancia que tiene el mantenerse activo en esta población, para así evitar los deterioros físicos y mentales a consecuencia del COVID-19. Esto se refuerza con los hallazgos del estudio de James $\mathrm{E}$ et al. $^{32}$, quienes demostraron que el ejercicio logra potenciar la función inmune, al prevenir y / o retrasar la inmunosenescencia, fortaleciendo así el sistema inmune.

En el estudio de Nieman et al. ${ }^{33}$, se determinó que la realización de actividad física de intensidad moderada durante el curso de infecciones respiratorias es segura y genera beneficios siempre y cuando las personas no presenten síntomas respiratorios mayores o fiebre. Cabe destacar también que en este mismo estudio se determinó que realizar ejercicio de alta intensidad durante la infección se asocia a un aumento de complicaciones respiratorias ${ }^{33}$.

El lograr combatir adecuadamente la infección por SARS-CoV-2, dependerá principalmente por el estado inmunitario de la persona. Se postula que el éxito final frente a la infección dependerá de factores genéticos, de la exposición anterior a otros virus y del nivel de acondicionamiento físico de la persona ${ }^{11}$. Lamentablemente, existe escasa evidencia en relación a los efectos de la actividad física sobre el sistema inmune en el caso de curso activo de infección por SARS$\mathrm{CoV}-2$, y las indicaciones se basan más en ejercicios para fortalecer la musculatura respiratoria y evitar deterioro del $\mathrm{VO}_{2}$ máximo en los pacientes contagiados $^{8}$.

Una de las interrogantes que aún no ha sido resuelta es qué sucede con el sistema inmune en caso de que nos infectemos por SARS-CoV-2. ¿Seremos capaces de desarrollar inmunidad? Y si lo hacemos, ¿Por cuanto tiempo estaremos protegidos? La respuesta es aún desconocida. Sin embargo ya se han evidenciado numerosos casos de reinfección por SARS-CoV-2 ${ }^{34}$.

\section{Prescripción de ejercicio en tiempos de pandemia}

Antes de describir la prescripción de ejercicio en tiempos de pandemia, es fundamental mencionar que toda persona sana; ya sea niño, adulto o persona mayor puede realizar actividad física. Lo mismo aplica en aquella población que tiene alguna afección crónica de salud, siempre y cuando su condición de salud de base se encuentre controlada y cuente con autorización de su médico tratante ${ }^{35}$. Distintos autores han resaltado la importancia de mantenernos activos, para evitar el deterioro de todo nuestro organismo y enfrentar de mejor manera esta pandemia ${ }^{13,31,36,37}$. Es por esto que especificaremos algunas recomendaciones generales de actividad física para mantenernos activos y con un sistema inmune preparado.

\section{Recomendaciones de actividad física en niños}

Respecto de las recomendaciones de la OMS en menores de un año y preescolares, se indica que se mantengan lo más activos posibles, con actividades lúdicas de forma libre y programada .Se recomienda que en los niños de edad preescolar entre 3 y 4 años se realicen, a lo menos, 60 min diarios de actividad física de moderada a alta intensidad. Y para aquellos niños en el rango de 4 a 6 años de edad, lo mínimo recomendado es 180 min diarios de actividad física ${ }^{38}$.

Para niños mayores de 6 años y adolescentes, la recomendación es que realicen al menos 60 min diarios de actividad física de moderada a alta intensidad. Ellos además deben realizar ejercicios, al menos, 3 veces a la semana que impliquen fuerza muscular ${ }^{38}$.

\section{Adultos y adultos mayores}

Para los adultos mayores a 18 años y menores de 60, las recomendaciones generales son las de realizar ejercicio por un mínimo de $150 \mathrm{~min}$ a la semana. Estas actividades deben ser de moderada intensidad, o bien, $75 \mathrm{~min}$ de actividad física de alta intensidad ${ }^{38}$. Se pueden implementar programas de ejercicios sencillos en el hogar, como caminatas dentro de la casa y ejercicios de fortalecimiento utilizando sillas $u$ otros implementos que nos permitan mantener nuestro estado físico, incluyendo además ejercicios de elongación y equilibrio ${ }^{37}$.

Para los adultos mayores a 60 años, queremos recalcar la importancia de la actividad física, ya que no solo muestra beneficios en los estados crónicos de alguna enfermedad presente, sino que también tiene efectos adicionales sobre las condiciones propias del envejecimiento, entendiéndose que el ejercicio afecta positivamente en prevenir fragilidad, sarcopenia/dinapenia, riesgo de caídas, autoestima y deterioro funcional o cognitivo ${ }^{39}$.

La OMS recomienda $150 \mathrm{~min}$ ejercicio de intensidad moderada a la semana; agregando ejercicios de fuerza focalizados 3 veces a la semana para prevenir riesgo de caídas en aquellos adultos mayores con poca movilidad ${ }^{38}$.

Jiménez-Pavón et al. ${ }^{31}$, describen en su investigación cómo afecta la cuarentena por COVID-19 
a las personas adultas mayores y concluyen que es fundamental mantenerse activos. Los autores recomiendan, de forma más enfática que la OMS, que esta población debe realizar entre 5-7 veces por semana ejercicio aeróbico, sumando entre 200 y $400 \mathrm{~min}$ de actividad física aeróbica a la semana. Además, se debe agregar ejercicios de fuerza 2-3 veces por semana. Las intensidades de trabajo recomendadas varían entre $40-60 \%$ de la frecuencia de reserva o $65-75 \%$ de la frecuencia cardiaca máxima ${ }^{31}$.

\section{Quiebre en la conducta sedentaria como una estrategia para la población en general}

La realización de quiebres en la conducta sedentaria debe considerarse como una estrategia simple para evitar el deterioro que genera, en especial sobre los niveles de estrés y sistema inmune $^{38}$. "Conducta sedentaria" se define como cualquier actividad con bajo costo energético (1,0 a 1,5 Mets.), que ocurre durante las horas de vigilia como, por ejemplo, estar sentado o $\operatorname{acostado}^{38}$. Esta conducta es la que encontramos en la condición actual de confinamiento a espacios pequeños y teletrabajo y que está asociada a enfermedades metabólicas, depresión del sistema inmune y mayor riesgo de muerte ${ }^{40-42}$.

Un ejemplo de rutina sencilla para aplicar, realizable en todo rango de edades, es el protocolo implementado por Dempsey et al. ${ }^{41}$, éste ha demostrado beneficios metabólicos importantes y consiste en detener la actividad sedentaria cada $45 \mathrm{~min}$ (o $60 \mathrm{~min}$ ) y realizar $3 \mathrm{~min}$ de ejercicio dividido de la siguiente manera: $1 \mathrm{~min}$ de flexoextensión de tobillo en el lugar, 1 min de sentadillas en el lugar (profundidad de la sentadilla a tolerancia y en caso de adultos mayores pueden remplazarse por 1 minuto parándose y sentándose de una silla con apoyo de los brazos), 1 minuto de caminata en el lugar.

\section{Conclusiones}

La actividad física y el ejercicio son herramientas no farmacológicas con evidencia científica robusta que podrían contribuir a disminuir la probabilidad de contagio de la población en general, así como en la población que presenta comorbilidades, inmunosenescencia y otros factores de riesgo que se han asociado con presentaciones más agresivas de esta enfermedad.

Se enfatiza sobre la importancia de realizar las dosis de ejercicio físico en base a las recomendaciones entregadas, para así evitar el deterioro que produce la inactividad física, aumen- tada por el confinamiento a espacios reducidos que estamos viviendo debido a las medidas de restricción.

Es fundamental seguir investigando los efectos de la actividad física en la prevención, manejo, pronóstico y recuperación de los pacientes con COVID-19.

\section{Biliografía}

1.- World Health Organization. Statement on the second meeting of the International Health Regulations. Emergency Committee regarding the outbreak of novel coronavirus (2019-nCoV). https://www.who.int/ news-room/detail/30-01-2020-statement-on-the-secondmeeting-of-the international-health-regulations-(2005)emergency-committee-regarding-the-outbreak-of-novelcoronavirus-(2019-ncov) (Consultado 31/03/2020).

2.- World Health Organization. Novel Coronavirus (2019nCoV): Situation Report-19. https://www.who.int/ emergencies/diseases/novel-coronavirus-2019/situationreports/(Consultado 30/03/2020).

3.- Coronavirus Update. https://www.worldometers.info/ coronavirus/ (Consultado 03/04/2020).

4.- THIRUMALAISAMY P, VELAVAN C. MeyerFirst. The COVID-19 epidemicpublished: 12 February 2020 https://doi.org/10.1111/tmi.13383.

5.- OMS | Inactividad física: un problema de salud pública mundial. Who.int. 2019.https://www.who.int/dietphysicalactivity/factsheet_inactivity/es. (Consultado el 31/03/2020).

6.- MARQUES A, SANTOS T, MARTINS J, MATOS MG, VALEIRO MG. The association between physical activity and chronic diseases in European adults. Eur J Sport Sci 2018; 18 (1): 140-9.

7.- VELAVAN TP, MEYER CG. The COVID-19 epidemic. Trop Med Int Health 2020; 25 (3): 278-80.

8.- CASCELlA M, RAJNIK M, CUOMO A, DULEBOHN S, DI NAPOLI R. Features, Evaluation and Treatment Coronavirus (COVID-19) In: StatPearls. Treasure Island (FL). https://www.ncbi.nlm.nih.gov/ books/NBK554776/. (Consultado el 30/04/2020).

9.- $\quad$ LIU Z, BING X, ZA ZHI X. The Epidemiological Characteristics of an Outbreak of 2019 Novel Coronavirus Diseases (COVID-19) in China 2020; 41 (2): 145-51.

10.- FISHMAN JA, GROSSI PA. Coronavirus-19 (COVID-19) in the Immunocompromised. Am J Transplant 2020; 20 (7): 1765-67.

11.- CHEN N, ZHOU M, DONG X, QU J, GONG F, HAN $\mathrm{Y}$, et al. Epidemiological and clinical characteristics of 99 cases of 2019 novel coronavirus pneumonia in Wuhan, China: a descriptive study. Lancet 2020; 395 (10223): 507-13.

12.- CAO J, TU WJ, CHENG W, YU L, LIU JK, HU X, LIU Q. Clinical Features and Short-term Outcomes of 
102 Patients with Corona Virus Disease 2019 in Wuhan, China. Clin Inf Dis 2020; ciaa243: 1-21.

13.- LUZI L, RADAELLI MG. Infuenza and obesity: its odd relationship and the lessons for COVID19 pandemic. Acta Diabetologica 2020; 5: 1-6.

14.- STREWE C, MUCKENTHALER F, FEUERECKER M, YI B, RYKOVA M, KAUFMANN I, et al. Functional changes in neutrophils and psychoneuroendocrine responses during 105 days of confinement. J Appl Physiol 2015; 118 (9): 1122-7.

15.- SHIMAMIYA T, TERADA N, HIEJIMA Y, WAKABAYASHI S, KASAI H, MOHRI M. Effects of 10-day confinement on the immune system and psychological aspects in humans. J Appl Physiol 2004; 97: 920-4.

16.- BOWDEN KA, PICKLES S, SPRUNG VS, KEMP GJ, ALAM U, MOORE DR, et al. Reduced physical activity in young and older adults: metabolic and musculoskeletal implications. Ther Adv Endocrinol Metab 2019; 10: 1-15.

17.- LIGHTFOOT JT. Current Understanding of the Genetic Basis for Physical Activity. J Nutrition 2011; 141 (3): 526-30.

18.- MORRIS JN, CRAWFORD MD. Coronary Heart Disease and PhysicalActivityOfWork: Evidenceof a National Necropsy Survey. Brit Med J 1958; 20 (12): 1445-96.

19.- RABØL R, PETERSEN KF, DUFOUR S, FLANNERY C, SHULMAN GI. Reversal o fmuscle insulin resistance with exercise reduces postprandial hepatic de novo lipogenesis in insulin resistant individuals. Proc Natl Acad Sci USA 2011; 108: 13705-9.

20.- MORTON AB, SMUDER AJ, WIGGS MP, et al. Increased SOD2 in thediaphragmcontributestoexerciseinducedprotectionagainstventilator-induceddiaphragmdysfunction. Redox Biol 2019; 20: 402-13.

21.- SMUDER AJ, MIN K, HUDSON MB, et al. Enduranceexerciseattenuatesventilator-induceddiaphragmdysfunction. J Appl Physiol 2012; 112 (3): 501-10.

22.- SMUDER AJ, MORTON AB, HALL, S E, et al. Effects of exercise preconditioning and HSP72 on diaphragm muscle function during mechanical ventilation. J Cachexia Sarcopenia Muscle 2019; 10 (4): 767-81.

23.- SOLLANEK KJ, BURNISTON JG, KAVAZIS AN, et al. Global Proteome Changes in the Rat Diaphragm Induced by Endurance Exercise Training. PLoS One 2017; 12 (1): e0171007.

24.- ABALOS KC, PETRI WA. Infectious disease and sports. In: Miller MD, Thompson SR, eds. DeLee\&Drez'SOrthopaedic Sports Medicine. 4th ed. Philadelphia, PA: Elsevier Saunders; 2015: chapter 20.

25.- LANFRANCO F, GHIGO E, STRASBURGER CJ. Hormones and athletic performance. In: Melmed S, Polonsky KS, Larsen PR, Kronenberg HM, eds. Williams Textbook of Endocrinology. 13th ed. Philadelphia, PA: Elsevier; 2016; chapter 26.

26.- LOWDER T, PADGETT DA, WOODS JA. Moderate exercise protects mice from death due to influenza virus. Brain Behav Immun 2005; 19 (5): 377-80.

27.- WARREN KJ, OLSON MM, THOMPSON NJ. Exercise Improves Host Response to Influenza Viral Infection in Obese and Non-Obese Mice through Different Mechanisms. PLoS One 2015; 10 (6): e0129713.

28.- SIM YJ, YU S, YOON KJ, LOIACONO CM. Chronicexercise reduces illness severity, decreases viral load, and results in greater anti-inflammatory effects than acute exercise during influenza infection. $J$ Infect Dis 2009; 200 (9): 1434-42.

29.- ROONEY BV, BIGLEY AB, LAVOY EC, et al. Lymphocytes and monocytes egress peripheral blood within minutes after cessation of steady state exercise: A detailed temporal analysis of leukocyte extravasation. Physiol Behav 2018; 194: 260-7.

30.- ADORE EL, SÁEZ DE ASTEASU ML, IZQUIERDO M. Multicomponent exercise and the hallmarks of frailty: Considerations on cognitive impairment and acute hospitalization. Exp Gerontol 2019; 122: 10-4.

31.- JIMÉNEZ-PAVÓN D, CARBONELL-BAEZA A, LAVIE CJ. Physical exercise as therapy to fight against the mental and physical consequences of COVID-19 quarantine: Special focus in older people. Prog Cardiovasc Dis 2020; 63 (3): 386-8.

32.- TURNER JE. Is immunosenescence influenced by our lifetime "dose of exercise? Biogerontology 2016; 17: 581-602.

33.- NIEMAN DC, JOHANSSEN LM, LEE JW, ARABATZIS K. Infectious episodes in runners before and after the Los Angeles Marathon. J Sports Med Phys Fitness 1990; 30 (3): 316-28.

34.- OTA M. Will we see protection or reinfection in COVID-19? Nat Rev Immunol 2020; 20 (6): 351.

35.- JAKOBSSON J, MALMC, FURBERG M, EKELUND U, SVENSSON M. Physical Activity During the Coronavirus (COVID-19) Pandemic: Prevention of a Decline in Metabolic and Immunological Functions. Front Sports Act Living 2020; (2): 10.3389/ fspor.2020.00057.

36.- NGUYEN HC, NGUYEN MH, DO BN, TRAN CQ, NGUYEN TT, PHAM KM, et al. People with Suspected COVID-19 Symptoms Were More Likely Depressed and Had Lower Health-Related Quality of Life: The Potential Benefit of Health Literacy. J Clin Med 2020; 9(4): 965.

37.- CHEN P, LIJUAN MAO L, NASSIS G, HARMER P, AINSWORTH B, LI F. Coronavirus disease (COVID-19): The need to maintain regular physical activity while taking precautions J Sport Health Sci 2020 Mar; 9 (2): 103-4.

38.- Organización Mundial de la Salud, (OMS). How do I stay active in and around the home? https://www.who. $\mathrm{int} /$ news-room/q-a-detail/be-active-during-covid- 19 . (Consultado en 01/04/2020)

39.- CADORE EL, SÁEZ DE ASTEASU ML, IZQUIER- 
DO M. Multicomponent exercise and the hallmarks of frailty: Considerations on cognitive impairment and acute hospitalization. Exp Gerontol 2019; 122: 10-4.

40.- CRISTI-MONTERO C. Considerations regarding sedentary and physical inactivity. Aten Primaria 2016; 48 (5): 341.

41.- DEMPSEY PC, DUNSTAN DW, LARSEN RN,
LAMBERT GW, KINGWELL BA, OWEN N. Prolonged uninterrupted sitting increases fatigue in type 2 diabetes. Diabetes Res Clin Pract 2018; 135: 128-33.

42.- OWEN N, SPARLING PB, HEALY GN, DUNSTAN DW, MATTHEWS CE. Sedentary behavior: emerging evidence for a new health risk. Mayo Clin Proc 2010; 85: 1138-41.

Correspondencia a:

Astrid von Oetinger

Escuela de Kinesiología, Facultad Ciencias de la Salud,

Universidad San Sebastián, Lota 2464,

Providencia, Santiago, Chile.

Email: astridvon@gmail.com 